\title{
DE ESCRIBIR Y DESCRIBIR
}

\author{
Carmen de Castro Castro \\ Universidad de Córdoba
}

Recibido: 5 junio 2011 / Aceptado: 8 octubre de 2011

ISNN: $1697-7467$

\begin{abstract}
RESUMEN: Nuestro estudio pretende mostrar la situación de la escritura en L.E. al comienzo de la Universidad y, en particular, en los textos descriptivos en lengua francesa. Partimos de una encuesta y de 20 textos que forman el corpus de trabajo. Vemos que los alumnos no dominan la competencia textual ni describen lo que ven y sienten, sino que se limitan a reproducir esquemas de ejercicios gramaticales conocidos. Sus textos carecen de organización y son pobres en actualizadores, conectores y vocabulario. Proponemos modificar las situaciones de escritura en el aula, seguir un guión de corrección (tanto para el alumno como para el profesor) y dedicar un mayor espacio a la escritura.
\end{abstract}

Palabras clave: descripción, escritura, corrección, competencia textual

\section{About writing and description}

ABSTRACT: The goal of our study is to show our University students' level of writing in a Second Language at the initial stage of their University period, particularly regarding French descriptive texts. Our working corpus is formed by a questionnaire plus twenty texts. We can state that our students neither command text competence, nor they describe what they see and feel, but they rather reproduce familiar patterns for exercises. Their texts lack organisation, they show few connectors, little vocabulary and are poorly actualised.

Our proposal it to modify writing situations in the classroom, to follow a pattern for marking the exercises (for both students and teachers), and finally, to devote more time to writing exercises.

Keywords: description, writing, marking, text competence.

\section{INTRODUCCIÓN}

Aún queda mucho camino por andar antes de ver a los alumnos, que ingresan en la Universidad, con un nivel adecuado en la escritura, más aún, si pretendemos que sean capaces de expresar lo que ven, lo que ocurre o lo que sienten en lengua extranjera.

A pesar del corriente uso de terminología pragmática que encontramos en los actuales manuales de FLE, la competencia textual tiene poco peso en las actividades que se proponen. En la descripción, se suelen utilizar esquemas muy cerrados, en los que prácticamente se substituye una palabra por otra, de manera obligatoria, o bien, el texto es una simple contextualización de un campo semántico muy minucioso y de poca aplicación. Todo ello provoca una dificultad para transformar lo susceptible de descripción en palabras; el alumno recurrirá a lo que ha aprendido a decir en francés, aunque no se ajuste totalmente con lo que desearía describir. 


\section{Evaluación INICIAL Y CORPUS DEL TRABAJO}

Nuestra elección ha sido motivada por ser la descripción el tipo de texto que, al parecer, "ha sido más trabajado" y es "más fácil de realizar" según una encuesta realizada a 56 alumnos de bachillerato y a 25 alumnos de primer curso de Universidad sobre actividades de escritura, corrección y tipos textuales.

\section{CUESTIONARIO SOBRE ACTIVIDADES DE ESCRITURA Y CORRECCIÓN}

1. ¿Cuantas actividades de redacción, que hayan sido corregidas, has realizado entre los dos cursos de Bachillerato?

\begin{tabular}{|l|l|}
\hline - Menos de 4 & 5 \\
\hline - Entre 4 y 6 & 24 \\
\hline - Entre 6 y 10 & 25 \\
\hline - Entre 10 y 15 & 14 \\
\hline - Más de 15 & 1 \\
\hline
\end{tabular}

2. ¿Que extensión tenía la mayor parte de las redacciones?

\begin{tabular}{|l|l|}
\hline - Menos de 50 palabras & 17 \\
\hline - Entre 50 y 80 palabras & 30 \\
\hline - Entre 80 y 150 palabras & 18 \\
\hline - Entre 150 y 200 palabras & 2 \\
\hline - Más de 200 palabras & 2 \\
\hline
\end{tabular}

3. ¿Que tipo de texto es el que más has trabajado?

\begin{tabular}{|l|l|}
\hline - El relato personal en pasado & 10 \\
\hline - La carta informal & 7 \\
\hline - El artículo periodístico & 5 \\
\hline - El comentario y la argumentación & 11 \\
\hline - El cuento & 4 \\
\hline - El relato de ficción & 4 \\
\hline - El diálogo & 43 \\
\hline - La descripción & 41 \\
\hline
\end{tabular}

${ }^{1}$ Desde nuestro punto de vista, la descripción es un tipo de texto difícil de trabajar de manera aislada, y no debería estudiarse si no es dentro de un contexto que lo justifique (ver propuestas). 
4. ¿Cuál te resultaba más fácil de redactar?

\begin{tabular}{|l|l|}
\hline - El relato personal & 23 \\
\hline - La carta informal & 6 \\
\hline - El artículo periodístico & 5 \\
\hline - El comentario y la argumentación & 6 \\
\hline - El cuento & 14 \\
\hline - El relato de ficción & 13 \\
\hline - El diálogo & 36 \\
\hline - La descripción & 40 \\
\hline
\end{tabular}

5. La actividad de redacción se realizaba:

\begin{tabular}{|l|l|l|}
\hline - De forma libre individual como tarea para casa & 12 & 25 \\
\hline - Se comenzaba en la clase y se continuaba en casa & 4 & 28 \\
\hline $\begin{array}{l}\text { - Formaba parte de la Unidad Didáctica, y se hacía en parejas durante el } \\
\text { tiempo de clase. }\end{array}$ & 1 & 28 \\
\hline - Se dedicaban sesiones específicas para redactar, “Taller de escritura” & 1 & \\
\hline
\end{tabular}

6. Normalmente la redacción se corregía:

\begin{tabular}{|l|l|l|}
\hline - Por el profesor en su casa sobre la redacción en limpio & 9 & 43 \\
\hline - Por el profesor en su casa sobre borradores & 1 & 5 \\
\hline - Por el profesor en clase sobre borradores & 2 & 5 \\
\hline - El profesor corregía en la pizarra alguna de las redacciones & 5 & 9 \\
\hline - Por los compañeros antes de presentar la versión definitiva & 1 & 4 \\
\hline $\begin{array}{c}\text { - Por el propio alumno con ayuda de programas de tratamien- } \\
\text { to de textos, correctores y diccionarios }\end{array}$ & 1 & 4 \\
\hline
\end{tabular}

Del análisis de las respuestas a este cuestionario, nos gustaría subrayar, respecto de los items 1 y 2, la escasez de actividades de escritura realizadas entre los dos últimos cursos de bachillerato y la brevedad de los mismos, lo que justificaría, de manera general, el escaso dominio de la expresión escrita a este nivel.

Dentro de la experiencia metodológica, recogida en los items 5 y 6 , vemos una diferencia entre el grupo de la Universidad (en blanco) y el de Bachillerato (en gris). La tendencia se mantiene en el item 6, ya que, en ambos casos, en un alto porcentaje, es el profesor el que corrige las redacciones definitivas en su casa. 
Por el contrario, en el item 5, el grupo más homogéneo y con una metodología más reciente (dos grupos de Bachillerato) afirmaba que se trabajaba más a menudo la escritura durante la clase $y$, en menor proporción, como tarea en casa; lo que supone una diferencia respecto del otro grupo. Nos llama la atención, sin embargo, ver que el trabajo sobre borradores era escaso, con lo que la escritura, si bien constituía una actividad algo guiada desde el aula, aún no había adquirido un peso suficiente en la metodología. ${ }^{2}$

Estos resultados casarían bien con un buen porcentaje de descripciones correctas, dado el nivel avanzado de aprendizaje de la lengua, no obstante, la experiencia que presentamos muestra lo contrario: en una prueba inicial de morfosintaxis para el primer curso de Maestro especialista en L.E. realizada por los alumnos en la Universidad — seis cursos de mediaencontramos textos excesivamente simples, de estructura pobre, que obedecían a un estereotipo descriptivo similar al de los primeros cursos de aprendizaje del idioma.

Nos ha sorprendido ver como, alumnos, que más tarde han desarrollado correctamente narraciones complejas al poderle dedicar trabajo y tiempo, se limitaban a reproducir unas estructuras básicas descriptivas ${ }^{3}$, que, en muchos casos no llegaban a formar un verdadero texto. Estaban habituados, al parecer, a unos criterios de evaluación centrados en la morfosintaxis de la frase, las concordancias y la cantidad del léxico empleado ${ }^{4}$;

El corpus de nuestro trabajo son estas 20 descripciones de una fotografía realizadas por alumnos del primer años de estudio de Maestro especialista en Lengua extranjeras. La foto (ver anexo) muestra tres actores que posan con exagerada actitud en una cafetería. Se trataba de una prueba, lo que limitaba el tiempo disponible a unos 10 minutos aproximadamente y los alumnos no disponían de ninguna posibilidad de consulta gramatical o léxica.

Hemos recogido, como textos de referencia, 4 descripciones de la misma fotografía realizadas por adultos no francófonos con buen nivel de francés tratando de reproducir similares circunstancias. (en adelante A, B, C, D,E)

Hemos seleccionado, así mismo, una producción colectiva posterior realizada tras el comentario negativo de los resultados de la prueba. El profesor trabajó oralmente, en lengua materna, la descripción de la foto, para, a continuación, organizar grupos de escritura que expresaran en francés la nueva descripción desde el punto de vista escogido por el grupo (Ver corrección del profesor).

${ }^{2}$ En el PEPELF (2007) los futuros pofesores deben conceder una mayor atención a las actividades de escritura, ya que deben ser capaces de.:

7 je peux aider les apprenants à planifier et à structurer des textes écrits (par exemple en se servant d'aide-mémoire, de canevas, etc)

8 je peux aider les apprenants à contrôler, mettre en forme et améliorer leurs propres travaux d'écriture, ainsi qu'à les analyser.

9 je sais utiliser l'évaluation mutuelle et le retour d'information afin d'aider au processus d'écriture.

10 je sais utiliser des techniques variées pour aider les apprenants à prendre conscience de la structure, de la logique et de la cohérence d'un texte, ainsi qu'à produire des textes en conséquence.

${ }^{3}$ Los alumnos utilizan unos esquemas muy simples que han aprendido siguiendo las directrices vigentes para la enseñanza del Francés.

${ }^{4}$ De ahí su esfuerzo en detallar, incluso inventándolos, los colores de una foto en blanco y negro, las prendas de vestir, el mobiliario o los objetos de la fotografía. 


\section{LA DESCRIPCIÓN ENTRE LA TEORÍA Y LA DIDÁCTICA}

En los últimos 30 años la descripción ha tenido varios momentos en los que ha suscitado gran interés: los años 80 con. Adam y Petitjean, los 90 con Hamon y los 2000 con Reuter entre otros; pero, desde siempre, tanto en la enseñanza de la lengua como en las prácticas de escritura ha sido objeto de análisis y trabajo, pudiéndonos remontar, en este caso, al uso de la Reórica como guía y método.

Por el tipo de descripción se han caracterizado algunos géneros literarios, le réalisme, le naturalisme o el del nouveau roman entre otros, géneros que, desde comienzos del S. XIX, han sido utilizados por las metodologías más culturalistas como modelos en la enseñanza de la descripción y la escritura.

Es reconocida en Francia la pedagogía tradicional de la composición francesa basada en un método realista: la observación por medio de los sentidos fomenta el desarrollo del vocabulario, ya que se intenta etiquetar cada objeto, ruido o gesto.

A partir de los años 70 este modelo se replantea y comienza la era de la lingüística textual con la participación activa de los fundadores de la revista Pratiques. Se establece los tipos textuales y se integra la narratología en el estudio de la descripción. Las secuencias descriptivas adquieren una forma arborescente a partir de un thème tître que se desarrolla y complica mediante el despliegue de parties y sous-parties

En los años 90, Hamon añade la noción de le descriptif como lugar textual en el que se amplifica o desarrolla una competencia esencialmente léxica y paradigmática y una competencia enciclopédica. Es decir, una memoria, la mathesis o saber sobre los objetos, sujetos, el mundo y el propio texto.

Por su parte, Reuter, incorpora al texto descriptivo su intención de darle al lector una mayor precisión del objeto descrito. La escritura, al tener en cuenta al destinatario, hace que éste reconozca o reconstruya el efecto descriptivo. Reuter distingue presencias descriptivas más o menos extensas distribuidas o detalladas en los tipos textuales, no necesariamente descriptivos y, con Apothéloz adopta la noción de recorrido que definirá el concepto de procedimiento descriptivo: el que describe debe recortar la imagen virtual, seleccionar los elementos fundamentales y ordenar las expresiones que va a utilizar siguiendo para ello un eje lateral, vertical o de planos yuxtapuestos, de manera que el lector pueda captar los efectos elegidos por él.

En la aplicación en mayor o menor grado de estas teorías en los manuales de enseñanza de la lengua (de las lenguas maternas sobre todo) dos tendencias suelen dominar las actividades descriptivas: un trabajo de vocabulario que a menudo adolece de una escasa visión de conjunto de la organización textual, o, por el contrario, un trabajo bastante completo de la organización del texto, con presencia de esquemas, el cual, sin embargo, carece del ejercicio necesario sobre las palabras. El alumno no ha llegado a tener una verdadera competencia descriptiva cuando está aprendiendo su L.M. ${ }^{5}$

\footnotetext{
${ }^{5}$ David Chevalier en su D.E.A «Description et interdisciplinarité : une autre modèle pour l'apprentissage de l'écriture» afirma, tras haber analizado varios manuales de enseñanza de la escritura en lengua materna: Il me semble que l'enseignant et ses élèves sont condamnés à un travail sans, ou si peu, de vue d'ensemble de l'organisation textuelle ou bien, à un travail assez poussé, sur l'organisation textuelle, et même peut-être trop cadré à mon goût, sans le travail nécessaire sur les mots pour décrire.
} 


\section{LA DESCRIPCIÓN de PERSONAS EN FLE}

En todas las metodologías se ha dedicado una lección, unidad o actividad a la descripción de personas y, no obstante, este tipo de texto (salvo en algunos manuales de escritura seguidores fieles de Petitjean y Adam) no suele ser realmente trabajado. Los textos que se presentan al comienzo de la lección como modelos no son empleados como tales, pues las actividades propuestas a los alumnos, eludiendo la composición del texto, consisten en simples yuxtaposiciones de frases y variaciones de género en el léxico de las partes del cuerpo, los colores y el vestido, en realidad un trabajo del vocabulario y de la morfosintaxis del grupo nominal. Estas actividades son a veces exhaustivas y el texto resultante no es el fruto del análisis minucioso de la realidad sino un ejercicio de transformación de frases, o, todo lo más, en un texto descontextualizado, acumulativo y muy simple. Pero, ¿qué autor de manual no aprovecharía la descripción de un personaje para contextualizar las partes del cuerpo, las prendas de vestir y los colores? La descripción en LE nace estereotipada y permanece así en la memoria del estudiante si no se trabaja para que deje de serlo.

\section{- Ejemplo 1}

\section{Décrire les personnes}

Le corps : la tête - le visage

Les cheveux, les yeux, les oreilles, le nez, la moustache, la barbe, la bouche, les dents.

Un bras, une main, un doigt, une jambe, un pied.

\section{Pour caractériser}

avoir les cheveux noirs, bruns, châtains, blonds - longs / courts ; avoir les yeux noirs, marrons, bleus ; être grand - de taille moyenne - petit ; mesurer 1,80m - faire 1,80m ; être gros / mince ; avoir le visage rond / allongé. (Girardet, J. Cridlig, J.M. Santillana, 1996: 66)

Si bien hallamos buenos textos didactizados descriptivos, empleados como modelos; a menudo, las actividades de escritura que se basan en ellos no trabajan ni la composición, ni la focalización, ni la modalización y sólo subrayan la necesidad de incluir la descripción física (muy escueta en el modelo, por otra parte)

\section{- Ejemplo 2.}

Écoutez et lisez.

Magali. - Tu sais, cet acteur... Renoir... Renard... machin, quoi.

Céline. - Qui? Je ne vois pas.

Magali. - Mais, si. Le grand, athlétique, la cinquantaine.

Il a eu beaucoup de succès même aux États Unis.

Il joue dans les fils de Luc Besson.

Céline. - Ah! , tu veux dire Réno? Jean Réno?

(...)

Pour décrire quelqu'un, on décrit :

Le physique, le caractère

- Il est de taille moyenne, il a les yeux verts, le nez pointu... 
- Il est athlétique, musclé, sportif

- Elle a l'air intelligent / vive / absente.

- Il / Elle est sensible / timide / gai(e) / calme / sérieux (euse)...

(Di Giura, Marcella; Beacco, 2007: 128)

\section{- Ejemplo 3}

\section{Parisiens, qui êtes-vous? \\ Gina}

Boulevard de Belleville, vous verrez certainement une petite femme aux cheveux gris qui parle à ses amis ou à sa chienne, Capucine. Dans le quartier, tout le monde l'appelle Gigi. Née en Italie en 1927, cette femme de ménage à la retraite est arrivée à Belle ville après la guerre. (...)

\section{Écrire}

\section{Portraits}

À la manière de l'article: Parisiens, qui êtes-vous? p. 22, écrivez un article qui fait le portrait, de deux ados. (...) Dans votre article, vous ferez une description physique de ces ados (la couleur des cheveux, le style de vêtements qu'ils portent, etc.)

(Menand, $2009: 22 ; 29$ )

En niveles avanzados no suele aparecer ya la descripción de manera aislada, sino formando parte de una tipología textual comunicativa, cuyo enfoque implica un uso más funcional de la lengua.

Écrivez une lettre à un ami(e) français(e) pour lui donner de vos nouvelles, lui raconter ce que vous faites dans votre pays et lui proposer de venir passer des vacances chez vous (environ 200 mots)

Paradójicamente esta actividad —que no parece la más idónea para incluir una descripción detallada sobre el aspecto físico-personal - está acompañada de un cuadro léxico muy preciso para desarrollar la descripción física:

- couleur des yeux (bleus, marron, noirs, vert clair...) - Cheveux (longs, courts, frisés, raides, bouclés, bruns, blonds, roux, gris, poivre et sel, chauve...) - forme du visage (rond, pointu, ovale, carré, triangulaire...) - traits particuliers du visage (barbe, moustache, tatouage, cicatrices, lunettes, piercing...) - vêtements.

paradoja que se mantiene en el ejemplo propuesto por los autores como posible respuesta a dicha actividad, en donde no se incluye ninguno de los términos léxicos propuestos.

Todo lo cual nos lleva a preguntarnos si no existe un cierto conflicto entre los contenidos que tradicionalmente se ha considerado que «deben estar en los manuales» y que «los alumnos deben conocer», y un enfoque mucho más pragmático en el que se debería dar cuenta o describir lo que se ve o se percibe, lo que llama la atención, lo que sugiere tal o cual imagen, cuando tal descripción sea pertinente. 
Nos llama la atención que los textos descriptivos no tengan ya tanto papel en los manuales de FLE, y que, sin embargo, el habito adquirido de enumerar características físicas, vestidos, tamaños y colores para caracterizar a las personas, esté tan vigente y disponible en los estudiantes; de tal manera que, éstos, sin dudarlo, lo desarrollarán en cuanto tengan delante una imagen y no se detendrán en detallar, sin embargo, aquel rasgo que les sorprende o que es esencial.

En la enseñanza de la LE se parte del hecho de que los alumnos ya han adquirido el modelo descriptivo en su propia lengua, por lo que sólo parece insistirse en acumular el léxico correspondiente al color, la apariencia física o el vestido como base de la descripción. Parece olvidarse el hecho de que las acciones también describen o que el uso de las comparaciones, metonimias, metáforas no está reñido con un nivel asequible de la lengua.

Tampoco se trabaja la inclusión del texto descriptivo en un todo textual, ya sea como pausa narrativa o como componente de un texto prescriptivo, pongamos por caso, lo que deja a este tipo textual sin anclaje: ¿Por qué? ¿Para qué? ¿Para quién describir? La pausa descriptiva encontraría toda su razón de ser en un relato, formando un todo homogéneo y un tipo de texto característico o, en el resto de tipos textuales, lo descriptivo puede repartirse tanto en el desarrollo de la acción, como en la argumentación o la prescripción ${ }^{6}$.

Aunque metodológicamente el aislamiento y simplificación de la descripción tienen validez en el nivel de lengua más elemental, no deberían dejar de reintegrarse en un todo textual lo antes posible, pues, de lo contrario, más que una función mimésica, que era lo pretendido, el texto descriptivo del alumno cumplirá únicamente con su función matética que expone el saber, que intenta mostrar lo que se conoce, lo recién adquirido.

\section{AnÁlisis del CORPuS: las deSCRIPCIONES de LA FOTOGRAFíA}

Tras estas consideraciones generales hemos querido profundizar en la situación real de una producción de textos descriptivos. Por ello, hemos analizado 20 textos de una prueba inicial de alumnos de primer curso universitario que consistía, como ya hemos señalado anteriormente, en la descripción de una fotografía.

Las descripciones presentaban abundantes errores de léxico, gramática, organización textual y cohesión, y, el profesor las había calificado de pobres y excesivamente simples. En nuestra opinión tales términos son difíciles de interpretar por los alumnos, motivo este, que nos ha llevado a realizar un análisis más detallado de los textos, en concreto sobre: la extensión, exactitud y riqueza léxica de sustantivos, adjetivos, adverbios y verbos y la construcción del texto descriptivo.

\footnotetext{
${ }^{6} \mathrm{La}$ description est un terme générique qui désigne les modes de présences textuelles du descriptif dans un texte ou un fragment plus ou moins étendu avec une composante descriptive dont l'effet est plus ou moins perceptible. Le descriptif est una composante de tout texte construisant un effet spécifique. Il répond à une intention plus ou moins précise dans une production écrite. (David, 2007: 30).
} 


\subsection{Estudio léxico}

\subsubsection{Enumeración de los personajes}

En las 20 redacciones de los alumnos encontramos que los términos, que casi con exclusividad, han sido empleados para describir a los personajes son:

$\begin{array}{lr}\text { - Homme } & 36 \\ \text { - Femme } & 50 \\ \text { - fille } & 5 \\ \text { - Personnes } & 15\end{array}$

Observamos una gran uniformidad en las descripciones, parece que siguen un patrón único. Los términos escogidos son muy generales e indeterminados, lo que indica una marcada falta de exactitud. En algunas de las descripciones, femme se repite 4 veces; femme y homme 5. La alta frecuencia de uso de términos tan imprecisos indica, por una parte, un deficiente manejo léxico, pues indudablemente conocen sinónimos o términos más específicos, y, por otra, una incapacidad de recurrir a los pronombres para agilizar el texto y evitar repeticiones.

En las descripciones de los expertos vemos variedad y riqueza léxica, ya que los personajes de la escena son nombrados con diferentes términos mediante anáforas y sinónimos; este procedimiento demuestra un dominio del vocabulario y la necesidad de crear un texto cohesionado. Observamos que, en tres de ellos, la interpretación de la imagen como una escena de ficción (cinematográfica o teatral) ha llevado a elegir términos específicos tales cómo: starlette, gangster, acteur.

A- clients, couple, membres, gangsters, starlette, servante, serveuse, une blonde, fille.

$B$ - personnages, compagne, serveuse, couple, photographe

$C$ - serveuse, client, personnage, compagnon, clients

$D$ - acteur, couple, serveuse, mari, fille client.

E- personne, acteurs, personnages, couple, serveuse, fiancé, accompagnant, client.

\subsubsection{Descripción de los personajes}

Los alumnos han optado mayoritariamente por limitarse a describir el aspecto más estereotipado y simple de los personajes encuadrándolos en une photo (15), como si de una foto de familia se tratase. La elección de los elementos parece seguir un canon: se destacan las prendas de vestir, curiosamente, 8 alumnos hablan de chemise, elemento que no parece evidente que haya que describir, pero que forma parte del vocabulario conocido y asociado a la descripción de personas. Los términos elegidos para diferenciar a las dos mujeres son cheveux (8) y lunettes (9)

Blonde (13) es el adjetivo más utilizado, que les sirve tanto para identificar a una de las dos mujeres como para destacar a la que está en primer plano. Brune (6) es el otro adjetivo que caracteriza a la mujer situada en un segundo plano. 
En cuanto a la elección de los demás colores: noir et blanc (11), (11) sabemos que obedece, únicamente, a la percepción de los colores de la fotocopia. No hay, por lo tanto, un verdadero esfuerzo en transmitir la realidad que se intuye, los colores no son los originales, y no habría que centrarse en ellos.

Los expertos, por su parte, incorporan aquellos aspectos que realmente les han llamado la atención y sólo ésos: ni los ojos ni los cabellos son realmente descritos. En cuanto al vestido, los sustantivos elegidos son mucho más específicos: A - tablier, diadème, lunettes. $\mathrm{B}$ - chapeau, coiffe, foulard, costume, cravate, pero mucho menos numerosos y sin constituir, ni el núcleo, ni lo más importante de la descripción.

\subsubsection{Descripción de la situación espacial}

Tanto en las descripciones de los alumnos como en la de los expertos uno o dos sustantivos sirven para describir la situación: café, bistrot, bar. Los objetos que están en el primer plano visual son los más minuciosamente descritos por los alumnos que conocen este vocabulario: lait, sucre, donnuts, café, y su valor proporcional es mucho mayor que el de los expertos.

\subsubsection{Descripción subjetiva}

Los expertos, interpretan la imagen y, por ello, utilizan un vocabulario consistente en sustantivos adjetivos y adverbios mucho más precisos y modalizados que refleja estados de ánimo y actitudes,:

A - une blonde toute riante, toute distraite... ; il a l'air d'un gangster... ; l'air égayé et nonchalant de la servante.

B - un homme victime entre deux femmes... ; la femme assise est le passé, la coutume, le déjà-vu, l'ennui... ; la servante est l'avenir souriant, l'aventure à portée de main, le nouveau...; La servante se tourne souriante et coquette vers un quatrième personnage, le photographe.

$\mathrm{C}$ - Café d'une allure assez élégante et bohème qui apparaît dans un état de grande tranquillité... ; reflet d'un sentiment de solitude et d'attente... ; les jeunes clients semblent être insérés dans une ambiance mélancolique... ; la serveuse reste débout donnant l'impression de couleur et de mouvement.

$\mathrm{D}$ - L'homme du couple regarde avec insolence la femme sérieuse et pensive... ; elle fait un geste de la main droite indiquant quelque chose comme "et voilà"

En los textos de los alumnos, por el contrario, casi no existe matización. Es muy escaso el uso de adverbios que modalicen los adjetivos a excepción de très (25) cuyo uso consideramos excesivo y no muy justificado en general. Casi podríamos afirmar que es el elemento escogido para personalizar la descripción. En algunas redacciones se llega a emplear, 3, 4 o 5 veces incluso:

...Les deux femmes sont très belles, mais l'homme est un peu moins jeune qu'elles.

La femme blonde est la serveuse dans un café. Elle a une sourire très belle et très 
grande... une femme brune avec lunettes et la bouche très belle... Elle a des mains très longues.

\subsubsection{El grupo verbal}

El número de verbos en los textos de los alumnos es de media 13, número alto, si tenemos en cuenta la longitud de las redacciones, unas 62 palabras por término medio. Vemos que las frases son muy cortas y en ellas se repiten muchos verbos de significado muy amplio como «est», «il y a», seguidos de «a» y «porte».

Se puede decir que hay prácticamente un tipo de esquema común:

- Enumeración de los personajes mediante: il y a

- Descripción de los personajes: sujeto + verbo être + adjetivo (en plural o singular)

- Detalles de esta descripción:

- posesión con el verbo avoir

- descripción del vestido con el verbo porter. bre)

- acciones: regarder (el hombre a la mujer rubia) y servir (la mujer rubia al hom-

En los textos de los expertos, el número de verbos es en proporción menor $\mathrm{y}$, aunque no hay gran variedad, pues se trata de un instante recogido en el que no hay mucha acción, si abundan las expresiones verbales modalizadas.

\subsection{Construcción del texto descriptivo}

Además de la notable diferencia morfológica encontrada entre los dos grupos de descripciones, tal distancia se hace mayor al centrarse el análisis en la composición del texto.

La descripción de una fotografía es un texto de singulares características, ya que la imagen suministra una enorme cantidad de información. El que describe la foto, selecciona de ella aquellos rasgos que desea transmitir, y sabe que la imagen ofrece al lector esos mismos signos, que se duplican, más muchos otros complementarios a su información. De tal manera que el autor del texto puede optar: o bien limitarse a enumerar y especificar los signos más sobresalientes, con lo que demostraría la extensión de su vocabulario, o bien, utilizando su conocimiento del mundo ${ }^{7}$, no sólo identificarlos sino compararlos e interpretarlos.

A nuestro parecer, la mayor parte de los alumnos que hicieron la descripción se quedaron en un primer nivel de significación, limitándose a destacar el aspecto evidente de los actores y, por lo tanto, no llegaron a construir un verdadero texto descriptivo que acompañase a la imagen.

... La femme porte des lunettes et a les cheveux longs. Ella a une sourire très belle et très grande. La première femme est mince avec les cheveux courts et blonds ...

...Une femme est débout et elle est très heureuse. L' autre femme est très sérieuse, elle porte des lunnetes et regarde la caméra. Il a les cheveux courts et bruns, il porte un costume à rayures blanches et une cravate ...

\footnotetext{
7 Término adoptado por el CDCR.
} 
Si tomamos como base la terminología utilizada por J. M. Adam, en contraste con las operaciones generales de «aspectualisation» $\mathrm{y}$ «qualification» usadas por los alumnos, en los textos de los expertos hallamos una mayor cantidad de»mise en relation» $\mathrm{y}$ «assimilation» que son aquellas que incluyen y contextualizan la descripción, así como operaciones de reformulación, que provocan una disminución del número de frases, un aumento de la fluidez del texto y una mayor cantidad de información que no duplica sino que completa la de la imagen.

A -... La serveuse toute distraite, elle n'est attentive qu'à sa pose.

Elle semble une fille de spectacle nocturne, une espèce de starlette...

B -... La femme assise est le passé, la coutume, le déjà vu, l'ennui.

- La servante est l'avenir souriant, l'aventure à portée de main...

C-... Un café d'une allure assez élégante et bohème, à la façon des cafés parisiens.

... La lumière, provenant de la rue n'arrive pas à toucher les personnages de l'image, reflet d'un sentiment de solitude et d'attente...

... La serveuse reste débout, donnant expression de couleur et de mouvement...

\subsubsection{Progresión y recuperación de la información}

L'absence d'apport d'information entraînerait une paraphrase perpétuelle ; l'absence de points d'ancrage renvoyant à $d u$ «déjà dit» amènerait à une suite de phrases qui, à plus ou moins long terme, n'auraient aucun rapport entre elles. (Combettes, 1986: 69)

En los siguientes ejemplos de los alumnos faltan conectores que establezcan la relación lógica de la información y los nexos gramaticales son equívocos. Los datos nuevos no descansan ni en datos ya conocidos, ni en aquellos utilizados en las frases precedentes. Los textos no fluyen, progresan a saltos.

1. Dans cette photo nous pouvons trouver que deux femmes et un garçon sont dans un café et l'employée sert le café. Celle-ci est très bavarde et ne s'occupe pas des clients. La femme de lunettes regarde très désolée

2. Dans la photo apparaissent deux femmes et un homme dans un bar, une femme est débout et elle est heureuse, elle a une conversation avec une autre personne, elle est l'employée de cette cafetéria.

L'autre femme voit la situation et elle pense à autres choses...

En el primer caso, no queda claro cuantos personajes hay en la foto; si tres o cuatro, ya que no se dice que una de esas femmes es l'employée. Mas adelante, se habla de clients pero, una vez más el lector no sabe cuantas personas hay en la sala, ya que no ha indicado que los clientes son l'homme y una de las deux femmes nombrados al comienzo. En cuanto a la mujer de las gafas, tampoco se ha mencionado que se trataba de uno de los clientes, ni que formaba parte del trío descrito al comienzo.

En el segundo ejemplo no hay tampoco una verdadera progresión de la información y ésta es incompleta. El lector no sabe cuantas personas hay en la foto ni quienes son. 
Al comparar estos ejemplos con uno de los de referencia vemos que en éste se pone especial interés en aclarar, desde el comienzo, el número de personajes y su función: Un café. Deux clients homme et femme. Une servante. On dirait le petit déjeuner. Mediante el uso de clients en la primera frase se determina la función de estos dos personajes. La utilización de servante incluye el género y su ocupación. El experto, no sólo ha especificado al lector el papel de cada una de las personas, sino que lo ha hecho de la manera más económica posible.

Como ya hemos comentado con anterioridad, en los textos de los alumnos no se modaliza el texto: la imagen obviamente ya muestra los personajes, relaciones y apariencia; Lo único que ellos han añadido, o más bien demostrado, es que saben enumerar esas características en francés, aportando una tras otra con un cierto grado de detalle. Pero la foto en cuestión presenta acciones, miradas, actitudes, poses que inducen a una interpretación y cuyo comentario hubiera sumado una observación nueva.

Sólo en algunos casos encontramos algunos intentos de expresar simultaneidad y duración de acciones:

....et elle est en arrossant le café dans la taisse de l'homme qui est asseyé en regardant la servante

o algún tipo de interpretación:

1- Sa gaieté peut être signal de qu'elle va avor un très bon jour, mais les autres personnes paraissent être très découragés, parce qu'ils doivent faire quelque chose qu'ils n'aiment pas, parce qu'ils n'ont pas bien dormi ou pour d'autre raison, il peut avoir plusieurs.

2- L'autre femme qui est avec l'homme le regarde jalousement

En los textos de los expertos, por el contrario, hallamos un rico uso de conectores que matizan y personalizan la descripción: comme; d'après; tout semble indiquer; tandis que; à son tour; ne... que; pourtant; indiquant; qui ... Así mismo encontramos numerosos verbos con valor modal que indican la actitud o la manera de pensar del que describe. En concreto, podemos subrayar la posición de incertidumbre ante la visión de una foto en la que se adivinan intenciones y actitudes.

A- On dirait, semble, paraît s'intéresser davantage, elle semble être

$\mathrm{B}$ - La scène admet tant $\mathrm{d}$ interprétations que des regards. On pourrait y voir. La compagne feint de ne pas s'en soucier. La serveuse feint de ne pas s'occuper. Les gestes semblent s'adresser à l'homme assis.

C - On dirait qu'elle ne prête pas l'oreille aux clients, semblant être le centre de tous les regards.

- Les jeunes clients semblent êtres insérés dans une ambiance mélancolique.

D- Il semble que l'époque est.....

- La serveuse semble regarder la salle.

E- D' après les habits des acteurs... tout semble indiquer.

Elle sourit et avec un geste de la main droite semble dire : " et voilà» 
La certeza de los textos de los alumnos contrasta con la incertidumbre de los de los expertos, que avanzan hipótesis sobre la situación reflejada en la imagen.

\section{Corrección del PROFESOR}

Volviendo a la evolución de nuestro estudio de caso, el profesor comentó el mal resultado de la prueba, tras lo cual, en un intento de reproducir el proceso y las estrategias de la composición escrita de la LM, pidió a los alumnos que observaran la fotografía y la comentaran oralmente en español. Se escogieron las mejores descripciones y, con esta referencia, se organizaron grupos de escritura. El resultado fue bastante diferente del de la redacción primera:

Dans cette photo nous voyons un café avec l'air américain des années quarante ou cinquante.

La serveuse, une blonde avec une position exagérée et théâtrale sert du café au monsieur qui est assis et qui l'observe attentivement, les deux sans regarder ce qu'ils font. A son côté s'assis une mademoiselle très sérieuse comme si elle était observé.

Realement tout le monde a une attitude très bizarre et peu naturelle.

\section{Conclusiones}

Los datos de las encuestas y la prueba de descripción nos muestran que los alumnos tienen escaso hábito y dominio para construir un texto dentro de las circunstancias habituales de clase. Falta, quizás, un poco más de conocimiento, pero, sobre todo, práctica de escribir.

Los alumnos no están acostumbrados, en lengua extranjera, a diseñar lo que tienen que decir, a decidir lo que es importante y seleccionar la manera mejor y más clara de decirlo.

Esas frases simples que «evitan» los errores son enemigas de un correcto tejido del texto. Los términos demasiado generales y habituales no dicen casi nada y empobrecen el escrito.

No es cierto que el texto descriptivo sea fácil, ni que se trabaje bastante en el Bachillerato: realizar actividades descriptivas, subordinadas al estricto uso léxico y morfológico, no conlleva saber seleccionar los rasgos pertinentes, ni escoger las expresiones adecuadas, ni lograr que la descripción produzca el efecto requerido en el lector.

La falta de trabajo habitual dificulta la organización y articulación de párrafos, hace que no estén disponibles ni actualizadores, ni deícticos, ni conectores que expresen el punto de vista del que escribe. 


\section{Propuestas}

Indudablemente para poder escribir un texto correcto en LE hay que practicar mucho. Todos los alumnos encuestados habían escrito muy poco, por lo que proponemos que se incluya en la planificación docente actividades específicas de escritura, independientes de la reutilización de los conocimientos adquiridos en la lección. Queremos insistir en la conveniencia de realizar actividades de escritura en el aula, individual o en pequeños grupos, trabajando sobre borradores y recibiendo una orientación y corrección del profesor. (Esta actividad puede estar acompañada del uso de software específico de escritura, con biblioteca de modelos de textos, bases de datos de léxico y asistentes de escritura) ${ }^{8}$

En nuestra opinión, en los manuales no hay suficientes textos que sirvan, además de para ser analizados, como modelo de escritura. Aunque ya no es necesario que los haya, puesto que en Internet encontramos un amplio abanico de descripciones, relatos breves, textos publicitarios, comentarios o argumentaciones a los que se accede libremente. Sugerimos, por ello, que antes de la escritura los alumnos realicen una búsqueda de distintos tipos de texto, los seleccionen y acepten como posibles modelos.

La descripción no es en general un tipo de texto independiente, por lo que, para que sea comprendido más fácilmente por los alumnos, debe encuadrase en una situación comunicativa que lo origine y fundamente. Al trabajarse en su contexto la descripción adquiere funciones distintas: en una publicidad serviría para convencer, en una narración para profundizar en el lugar o las características de los personajes, en un pie de foto para provocar un nuevo punto de vista, añadir información que no aparece en ella o interpretar lo que sí aparece. Describir una fotografía en la clase de LE exige una serie de consignas previas que justifiquen la construcción del texto que la acompaña, de otro modo, puede convertirse en un ejercicio más de vocabulario y gramática

En inscrivant la description dans un projet narratif long (exemple : l'écriture d'un roman), j'ai pu démontrer avec mes élèves l'enjeu du passage descriptif dans la cohérence narrative et j'ai résolu en partie le blocage lié au manque d'intérêt à priori pour ce type de texte. (David Chevalier; 1999: 35).

Al crear un espacio para la escritura, ya sea en el aula o fuera del horario escolar, se le debe prestar atención a la situación de comunicación creada entre el profesor- lector y los alumnos. En ella, el sentido debe primar sobre la forma, la riqueza y personalidad del texto, sobre las faltas de superficie. Al fin y al cabo, el profesor lee un texto firmado por un autor, no corrige uno más de los ejercicios del manual.

$\mathrm{Ni}$ el alumno ni el profesor deben distanciar el proceso de escritura en LE del de la LM. La elaboración de la trama de un texto es similar en ambas lenguas, por lo que recurrir parcialmente a la traducción, al esquema en LM, como paso previo hasta llegar a un mejor dominio lingüístico, puede ayudar a identificar el tipo textual, la organización del texto y su coherencia (los alumnos mejoraron sensiblemente sus textos al haber trabajado la descripción oralmente en LM)

\footnotetext{
${ }^{8}$ Por ejemplo, Gammes d'écriture, (Manguenot, 1996).
} 
La corrección debe hacerla el alumno; si se le señalan las faltas, sabrá mejorar su texto y eliminar muchas de ellas. Desde el primer nivel de aprendizaje de la LE, el alumno es capaz de elaborar textos y corregirse, eso sí, siguiendo una guía clara, elaborada por el profesor o disponible en propuestas didácticas especializadas. Los profesores, por su parte, tendrían que sistematizar y codificar mejor su corrección.

Toda corrección debe responder a lo que se pretende con la elaboración de un texto, por ello, en lo que a la escritura se refiere, no debe hacerse tanto énfasis en la gramática de la frase simple, sino atender también a la elección del léxico o el dominio de la competencia semántica. A título de ejemplo proponemos una corrección en varias fases:

En una primera se ayudaría a los alumnos a eliminar las faltas de ortografía, conjugación y concordancia gramatical, lo que dejaría un panorama más claro para poder identificar el entramado del texto y sus carencias.

En una segunda o tercera - 1 orden no es lo importante - se estudiaría el léxico para ver si el término escogido es el que mejor se ajusta o cabría utilizar otro más preciso, más concreto o que añadiera un matiz personal.

En otra fase, se haría ver la progresión del texto: cómo se le va añadiendo información nueva, y, al mismo tiempo, se sigue manteniendo la anterior, cuando es necesario. Se propondría el uso adecuado de los artículos definidos, los adjetivos y pronombres demostrativos - que se conocen pero no se saben utilizar en el texto- las oraciones de relativo y las comparativas para explicar y calificar, las temporales, para situar la acción, las adversativas y consecutivas para matizarla, por ejemplo.

Al mismo tiempo, o después, se revisaría la construcción del texto propiamente dicha, buscando disminuir el número de frases, valorando la puntuación, haciendo ver la facilidad del uso de la elipsis, de la reformulación, de la nominalización o la adjetivación, incluso. Recomendamos, a este respecto, las publicaciones del www.ccdmd (ver anexos) relativas a la tutoría de los alumnos en la expresión escrita (aunque haya que modificar la tipología de faltas, ya que los alumnos a los que va dirigida tienen el francés como lengua segunda).

\section{Bibliografía}

Adam, J.M. and Petitjean, A. (1989). Le texte descriptif. Paris: Nathan.

Apothéloz, D. (1998). «Eléments pour une logique de la description et du raisonnement spatial» in : Y. Reuter (éd.), La description. Théories, recherches, formation, enseignement. Villeneuve-d'Ascq: Presses Universitaires du Septentrion,15-31.

Combettes, B. (1986). «Introduction et reprises des éléments d' un texte» in Pratiques 49, Metz.: 69-84.

David Chevalier, M-C. (1999). Description et interdisciplinarité : un autre modèle pour l'apprentissage de l'écriture. Mémoire de D.E.A.Université Garles de Gaulle. Lille.

Desnoyers, A. (2002) Les grammaires. Centre collégial de développement de matériel didactique. Montréal CCDMD.

Di Giura, M. and Beacco, J.C. (2007). Alors ?. Paris: Didier.

Division des politiques linguistiques, Strasbourg (2001): Cadre européen de référence pour les langues. Paris: Conseil de l'Europe / les Editions Didier.

Dupleix, D. Bruno, Mègre. (2007). Production écrite Niveaux B1/B2. Paris: Didier 
Girardet, J. Cridlig, J.M. (1996). Panorama. Madrid: Santillana Clé International.

Hamon, Ph. (1993). Du descriptif. Paris: Hachette.

Izaute, F. and Differ, M. (2002). Matériel pour Tutorat. Centre collégial de développement de matériel didactique. Montréal: CCDMD.

Manguenot, Fr. (2000). "Contexte et conditions pour une réelle production d'écrits en ALAO» in Alsic, 2, 187-206.

Menand, R. (2009). Le Nouveau Taxi 2. Paris: Hachette.

Newby, D., Allan, R., Fenner, A., Jones, B., Komorowska, H., Soghikyan, K. (2007). Portfolio européen pour les enseignants en langues en formation initiale. Conseil de l'Europe

Petitjean, A. (2001). «La description scolaire au sécondaire (college) de 1960 à 19997», in pratiques, 109/110, 125-163.

Reuter, Y. (1999). «La description en questions» in Y. Reuter (ed.), La description. Lille: Presses universitaires du Septentrion.

Vourzay, M-H. (1998). «Jalons pour une histoire de la description socolaire: deux «modèles didactiques» de la rédaction/description pour le premier cycle de sécondaire» in Pratiques 99, Metz: 27-42.

\section{8- (2.p.) Décrivez :}

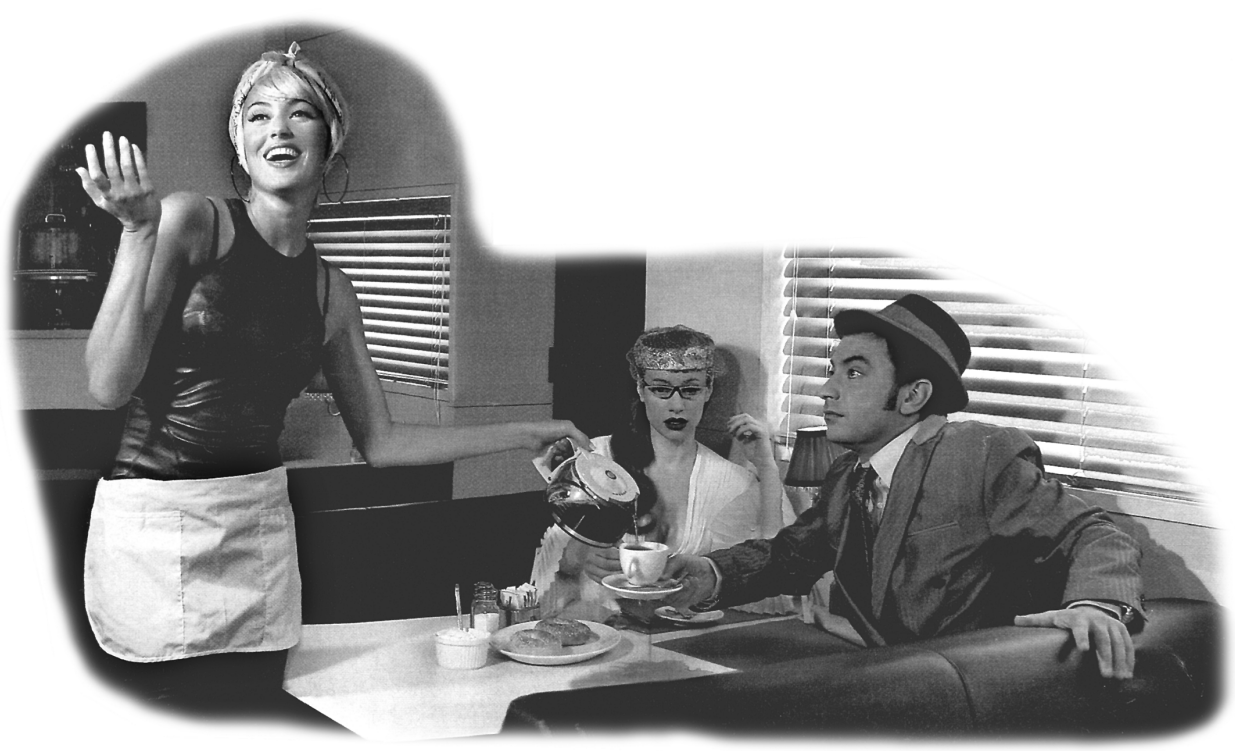




\section{PLAN DES ÉTAPES}

\begin{tabular}{|c|c|}
\hline & Fautes qui passent en rouge (encadrées) \\
\hline \multicolumn{2}{|l|}{ 1. La première rencontre } \\
\hline La démarche utilisée & $\begin{array}{ll}\text { O-1 } & \text { Orthographe d'usage } \\
\text { O-2 } & \text { Accents } \\
\text { O-3 } & \text { Homonymes (ex : amande, amende) }\end{array}$ \\
\hline \multicolumn{2}{|l|}{ 2. La phrase syntaxique autonome } \\
\hline $\begin{array}{l}\text { Reconnaissance du verbe conjugué (distinction } \\
\text { des différentes formes du verbe) }\end{array}$ & $\begin{array}{l}\text { O-3 Homonymie entre un verbe et un nom ou autre } \\
\text { chose : on/ont, son/sont, travail/travaille, à/a... } \\
\text { G-5 Conjugaison ou forme du verbe }\end{array}$ \\
\hline \multicolumn{2}{|l|}{ Distinction subordonnants/coordonnants } \\
\hline Découpage des phrases syntaxiques autonomes & P-1 Phrase sans verbe conjugué principal \\
\hline Ponctuation entre les P syntaxiques autonomes & P-3 Ponctuation entre les $P$ \\
\hline
\end{tabular}

\section{Les constituants de la phrase}

\begin{tabular}{l|ll}
\hline $\begin{array}{l}\text { Manipulations permettant de repérer le groupe } \\
\text { sujet et accord du verbe avec le sujet }\end{array}$ & $\begin{array}{l}\text { G-1 } \\
\text { P-1 }\end{array}$ & $\begin{array}{l}\text { Fautes d'accord du verbe conjugué principal } \\
\text { avence de l'un des constituants OBLIGATOIRES } \\
\text {-sujet ou prédicat }\end{array}$ \\
\hline $\begin{array}{l}\text { Identification des référents des pronoms sujets } \\
\begin{array}{l}\text { Manipulations permettant de repérer les } \\
\text { compléments de phrase }\end{array}\end{array}$ & P-3 & Problème de virgule liée au déplacement du CP \\
\hline $\begin{array}{l}\text { Ponctuation relative au déplacement des } \\
\text { compléments de phrase }\end{array}$ & P-3 & Problème de virgule entre le sujet et le prédicat \\
\hline $\begin{array}{l}\text { Ponctuation entre les constituants } \\
\text { Transformation de type et de forme }\end{array}$ & P-1 & $\begin{array}{l}\text { Problème de construction de la phrase } \\
\text { interrogative } \\
\text { Problème de construction de la phrase négative }\end{array}$
\end{tabular}

\section{Les groupes de mots}

Les différents types de groupes et les phrases subordonnées
P-4 Mauvais choix de pronom relatif

G-1 Fautes d'accord du verbe avec son sujet dans les phrases subordonnées
Les diverses possibilités de maturation syntaxique Construction des énumérations de groupes et énumérations dans les groupes
P-5 Problème de construction des énumérations de groupes (les prépositions à, en et de) et des énumérations

P-3 Problème de virgule lié à une phrase subordonnée ou à un groupe non essentiels

\section{Les principes régissant les accords}

Reconnaissance des donneurs et receveurs d'accord, ainsi que des mots appartenant à une catégorie invariable

Liens entre les donneurs et receveurs

\section{Le prédicat}

Reconnaissance des modificateurs du verbe, des compléments directs et indirects

Pronominalisation des compléments
G-2 Accord singulier / pluriel

G-3 Accord masculin / féminin ou avec être
G-4 Accord des participes passés employés seuls
P-3 Problème de virgule entre le verbe et ses compléments

G-4 Accord des participes passés employés avec avoir P-4 Mauvais choix de pronom complément du verbe 